\title{
Nonlinear Control of Electrodynamic Tether in Equatorial or Somewhat Inclined Orbits
}

\author{
Larsen, Martin Birkelund; Blanke, Mogens
}

Published in:

Proceedings of the 15th Mediterranean Conference on Control and Automation

Link to article, DOI:

10.1109/MED.2007.4433876

Publication date:

2007

Document Version

Publisher's PDF, also known as Version of record

Link back to DTU Orbit

\section{Citation (APA):}

Larsen, M. B., \& Blanke, M. (2007). Nonlinear Control of Electrodynamic Tether in Equatorial or Somewhat Inclined Orbits. In Proceedings of the 15th Mediterranean Conference on Control and Automation IEEE. https://doi.org/10.1109/MED.2007.4433876

\section{General rights}

Copyright and moral rights for the publications made accessible in the public portal are retained by the authors and/or other copyright owners and it is a condition of accessing publications that users recognise and abide by the legal requirements associated with these rights.

- Users may download and print one copy of any publication from the public portal for the purpose of private study or research.

- You may not further distribute the material or use it for any profit-making activity or commercial gain

- You may freely distribute the URL identifying the publication in the public portal 


\title{
Nonlinear Control of Electrodynamic Tether in Equatorial or Somewhat Inclined Orbits
}

\author{
Martin B. Larsen* and Mogens Blanke* SM-IEEE \\ *Automation at Oersted•DTU, Technical University of Denmark, DK 2800 Kgs. Lyngby, Denmark \\ mbl@oersted.dtu.dk and mb@oersted.dtu.dk
}

\begin{abstract}
This paper applies different control design methods to a tethered satellite system (TSS) to investigate essential control properties of this under-actuated and nonlinear system. When the tether position in the orbit plane is controlled by the tether current, out of orbit plane motions occur as an unwanted side effect, due to nonlinear interaction with the Earth's magnetic field. This paper focus on the uncontrollable out-of-plane motions and the robustness against B-field uncertainty associated with each of three popular controller design methodologies for nonlinear systems: linear quadratic feedback designed for the controllable subspace of the system, a feedback linearization design and a sliding mode control. The controllers are evaluated by their ability to suppress variations in the B-field and their robustness with respect to the internal dynamics.
\end{abstract}

\section{INTRODUCTION}

Space tethers have been studied over the last decades due to their potential of providing cheap actuators for spacecraft adjustment and altitude control (see e.g. [1]). Assuming a tethered satellite system (TSS) orbiting the Earth with an electrodynamic tether, the magnetic field of the Earth will induce a voltage along the tether. With an electrodynamic tether that is able to collect electrons from the ionosphere, a current will flow through the tether, which will give rise to a Lorentz force acting on the tether. The principle of electrodynamic tethers has mainly been investigated in connection to de-orbiting of obsolete satellites, but the principle can be utilized to perform any orbit adjustment. Having control of the current in the tether as control input, the tether system is under-actuated and the internal dynamics of the system is essential.

It is common to neglect the flexibility of tethers and use rigid body models. Such a model were used in [2] to investigate tether behavior in inclined orbits and new results regarding periodic solutions and their stability were found. Feedback controllers for a rigid tether model were investigated in [3], where a simple control scheme was proposed for control of tether position, based on the energy function of the system. In [4] periodic solutions were stabilized using time-delayed autosynchronization (TDAS). This control design assumed a constant current through the tether, while two control forces were added to the model. An energy rate feedback controller for stabilizing periodic solutions was designed in [5]. Ref. [6] considered the special case of a circular equatorial orbit and designed controllers based on the feedback linearized system, using a variable resistance to control the current through the tether.

1-4244-1282-X/07/\$25.00 @ 2007 IEEE
This paper uses the rigid tether model from [2] to investigate control strategies for the tether motion in the orbit plane, assuming a satellite in an equatorial or slightly inclined orbit. Most of the previous work with the rigid tether model used a nontilted dipole for modeling the magnetic field of the Earth. In this paper the nontilted dipole model will be expanded with uncertainty estimations based on a tilted dipole model. Three different controllers are investigated for their ability to suppress disturbance from the motions out of the orbit plane and their ability to handle uncertainties introduced in the magnetic field. The uncertainties in the magnetic field can be introduced by considering a tilted dipole model or by introducing a slightly inclined orbit.

\section{RIGID TETHER MODEL}

The TSS considered in this paper consists of a mainsatellite $\mathcal{B}$ with mass $m_{B}$ and a sub-satellite $\mathcal{A}$ of mass $m_{A}$ tethered with a rigid tether of length $l$ and mass $m_{t}$. Both satellites are model as point masses. It is assumed that the center of mass for the system coincides with the position of the main-satellite, i.e. $m_{B}>>m_{A}+m_{t}$. Furthermore it is assumed that the main-satellite is in a circular orbit of radius $R_{B}$ and orbit rate $\omega_{o}$. A current $I$ flows through the tether from $\mathcal{B}$ to $\mathcal{A}$. The tether is assumed to be subject to the Lorentz force and the gravity from the Earth, while the satellites are only subject to gravity. A sketch of the system is shown in Fig. 1. The

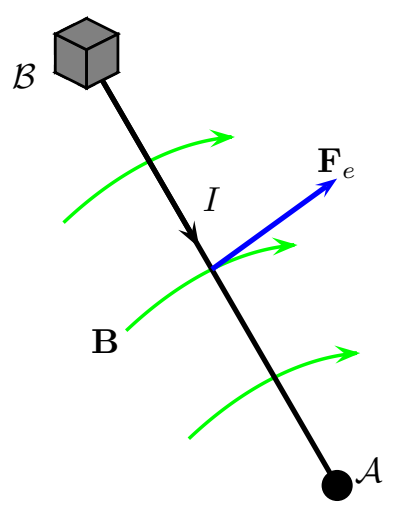

Fig. 1. Sketch of the tethered satellite system. The Lorentz force occur as the cross product between the tether position and the B-field.

tether position is described w.r.t. the main-satellite, hence a rotating frame centered at $\mathcal{B}$ is introduced with axes $\mathbf{x}_{o}$, $\mathbf{y}_{o}$ and $\mathbf{z}_{o}$. The vector $\mathbf{x}_{o}$ is defined along the geocentric 
position of $\mathcal{B}, \mathbf{z}_{o}$ is defined normal to the orbit plane, while $\mathbf{y}_{O}$ completes the right-handed coordinate system. $\mathbf{y}_{o}$ coincides with the velocity of the main-satellite due to the circular orbit assumption. The orbit frame can be seen i Fig. 2 along with the definition of the in-plane angle $\alpha$ and the out-of-plane angle $\beta$. The points along the tether

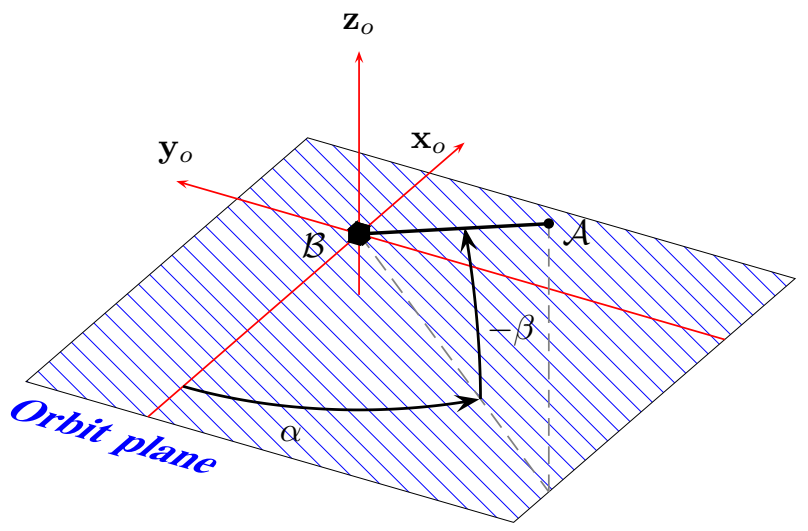

Fig. 2. Orbit frame description with the in-plane angle $\alpha$ and the out-of-plane angle $\beta$.

can be expressed in the orbit frame as a function of $\alpha$ and $\beta$

$$
\mathbf{r}(s)=s\left[\begin{array}{c}
-\cos \alpha \cos \beta \\
-\sin \alpha \cos \beta \\
-\sin \beta
\end{array}\right]
$$

where $s \in[0 l]$. Since the main-satellite is assumed in a circular orbit, it is natural to introduce the dimensionless time $\tau=\omega_{o} t$. Using $\tau$, the orbit period scales to $T=2 \pi$ and the true anomaly of the orbit can be written as $\theta=$ $\theta_{0}+\tau$, where $\theta_{0}$ is the initial position of the main-satellite.

\section{A. Lagrangian}

The Lagrangian of the system w.r.t. the orbit frame can be written as the difference between the kinetic energy and the total potential energy of the system

$$
\mathcal{L}=\mathcal{T}-\mathcal{V}
$$

where the $\mathcal{V}$ contains the gravitational potential from the Earth and the generalized potential originating from the inertial forces. Using $\alpha$ and $\beta$ as the generalized coordinates the Lagrangian can be written as [2]:

$$
\begin{aligned}
\mathcal{L}= & \frac{1}{6}\left(3 m_{A}+m_{t}\right) \\
& \omega_{o}^{2} l^{2}\left(\dot{\beta}^{2}+\cos ^{2} \beta\left((1+\dot{\alpha})^{2}+3 \cos ^{2} \alpha\right)\right)
\end{aligned}
$$

where $\left({ }^{\circ}\right)$ indicates the differentiation w.r.t. $\tau$.

\section{B. Generalized electrodynamic force}

The Lorentz force per unit length tether can be found as:

$$
\overline{\mathbf{F}}_{e}=I \hat{\mathbf{r}} \times \mathbf{B}
$$

where $I$ is the current through the tether, $\hat{\mathbf{r}}=\mathbf{r}(1)$ is a unit vector along the tether and $\mathbf{B}$ is the magnetic field. The current $I$ and the magnetic field $\mathbf{B}$ are assumed constant along the tether, i.e. independent of $s$. It is assumed that $I$ can be controlled without limitations. The generalized electrodynamic force $\mathbf{Q}=\left[\begin{array}{ll}Q_{\alpha} & Q_{\beta}\end{array}\right]^{T}$ can be found by projecting the Lorentz force per unit length tether onto the generalized coordinates and integrating along the tether

$$
\begin{aligned}
Q_{\alpha} & =\int_{0}^{l} \overline{\mathbf{F}}_{e} \cdot \frac{\partial \mathbf{r}(s)}{\partial \alpha} d s \\
Q_{\beta} & =\int_{0}^{l} \overline{\mathbf{F}}_{e} \cdot \frac{\partial \mathbf{r}(s)}{\partial \beta} d s
\end{aligned}
$$

which results in the following generalized force:

$$
\begin{aligned}
Q_{\alpha}= & \frac{l^{2}}{2} \cos ^{2} \beta \\
Q_{\beta} & =\frac{l^{2}}{2}\left(\tan \beta\left(\cos \alpha B_{x}+\sin \alpha B_{y}\right)-\sin \alpha B_{x}\right) I .
\end{aligned}
$$

\section{Equation of motion}

The dynamics of the tether can be found from the Lagrangian and the generalized force using Lagrange's equation

$$
\frac{d}{d \tau}\left(\frac{\partial \mathcal{L}}{\partial \dot{\mathbf{q}}}\right)-\frac{\partial \mathcal{L}}{\partial \mathbf{q}}=\mathbf{Q}
$$

where $\mathbf{q}=[\alpha \beta]^{T}$ is the generalized coordinates. Inserting (3) and (6) into (7) the model of the tether dynamics can be written as:

$$
\begin{gathered}
\ddot{\alpha}-2 \tan \beta(1+\dot{\alpha}) \dot{\beta}+\frac{3}{2} \sin 2 \alpha= \\
\Lambda\left(\tan \beta\left(\cos \alpha B_{x}+\sin \alpha B_{y}\right)-B_{z}\right) I \\
\ddot{\beta}+\frac{1}{2} \sin 2 \beta\left((1+\dot{\alpha})^{2}+3 \cos ^{2} \alpha\right)= \\
\Lambda\left(\cos \alpha B_{y}-\sin \alpha B_{x}\right) I
\end{gathered}
$$

where $\Lambda=\frac{3}{2\left(3 m_{A}+m_{t}\right) \omega_{0}^{2}}$. A more detailed derivation of the model can be found in [2] or in [4].

\section{Model OF THE MAGNETIC FIELD OF THE EARTH}

The magnetic field of the Earth can be modeled using a spherical harmonic model (see [7]). As a simplified model it is common to use a dipole model, which corresponds to a spherical harmonic expansion of degree one. The resulting dipole model is tilted compared to the rotational axis of the Earth, hence the B-field will vary as the Earth rotates. To keep the model independent of the rotation of the Earth, it is common to neglect the tilt of the dipole. This approach will also be used in this paper, but the uncertainty introduced by the approximation will be treated. Since dipole models are additive the tilted dipole can be viewed as the sum of three dipoles aligned with each of the axes in the rotating inertial frame. In the rotating inertial frame the $X$-axis crosses the longitude of Greenwich at equator, while the $Z$-axis coincides with the rotational axis of the Earth. The $Y$-axis completes the right-handed coordinate system, hence the $X Y$-plane 
denotes the equatorial plane. In recap, the dipole model can be written as:

$$
\mathbf{B}=\underbrace{\mathbf{B}_{Z}}_{\overline{\mathbf{B}}}+\underbrace{\mathbf{B}_{X}+\mathbf{B}_{Y}}_{\widetilde{\mathbf{B}}}
$$

where each term denotes a dipole model and the subscript indicates which axis it is aligned with. Since $\mathbf{B}_{Z}$ is independent of the rotation of the Earth it is natural to adapt this as the nominal model $\overline{\mathbf{B}}$, while the remaining dipoles denote the uncertainty $\widetilde{\mathbf{B}}$. Each of the dipole models can be written as:

$$
\mathbf{B}=\frac{R_{E}^{3} H}{R^{3}}(3(\hat{\mathbf{m}} \cdot \hat{\mathbf{R}}) \hat{\mathbf{R}}-\hat{\mathbf{m}})
$$

where $\hat{\mathbf{m}}$ is the dipole moment, $R_{E}$ is the radius of the Earth and $R_{E} H$ is the strength of the dipole. The coefficient $H$ is the Gauss coefficient from the spherical harmonic model. The 2005 value of the coefficients are shown in [7], while the relevant coefficients are repeated in Table I. The nominal B-field can be expressed in the

TABLE I

GAUSS COEFFICIENTS FOR IGRF MODEL OF DEGREE ONE.

\begin{tabular}{|c|r|c|}
\hline Coefficient & \multicolumn{1}{|c|}{ Value } & Dipole axis \\
\hline \hline$g_{1,0}$ & $-29556.8 \mathrm{nT}$ & $Z$ \\
\hline$g_{1,1}$ & $-1671.8 \mathrm{nT}$ & $X$ \\
\hline$h_{1,1}$ & $5080.0 \mathrm{nT}$ & $Y$ \\
\hline
\end{tabular}

orbit frame as a function of the orbit inclination $i$ and the true anomaly measured from the ascending node $\theta^{\prime}$ as:

$$
\overline{\mathbf{B}}=\frac{R_{E} H_{0}}{R^{3}}\left[\begin{array}{c}
-2 \sin \theta^{\prime} \sin i \\
\cos \theta^{\prime} \sin i \\
\cos i
\end{array}\right]
$$

where $H_{0}=\left|g_{1,0}\right|$. The corresponding expressions for the remaining dipoles can be found. These are, however, more comprehensive due to the rotation of the Earth, while only the upper bound will be stated here:

$$
\begin{aligned}
\left|\widetilde{B}_{x}\right| & \leq 2 \frac{R_{E}^{3}}{R^{3}} \widetilde{H}_{0} \\
\left|\widetilde{B}_{y}\right| & \leq \frac{R_{E}^{3}}{R^{3}} \widetilde{H}_{0} \\
\left|\widetilde{B}_{z}\right| & \leq \frac{R_{E}^{3}}{R^{3}} \widetilde{H}_{0}|\sin i|
\end{aligned}
$$

where $\widetilde{H}_{0}=\sqrt{g_{1,1}^{2}+h_{1,1}^{2}}$. The boundaries (12) can be found by a worse case estimate both w.r.t. the rotation of the Earth and the true anomaly. Under the circular orbit assumption the resulting B-field model can be written as:

$$
\mathbf{B}=\frac{R_{E}^{3} H_{0}}{R_{B}^{3}}\left[\begin{array}{c}
-2 \sin \theta \sin i+\widetilde{\Psi}_{x} \\
\cos \theta \sin i+\widetilde{\Psi}_{y} \\
\cos i+\widetilde{\Psi}_{z}
\end{array}\right]
$$

where the bound of the uncertainty terms $\widetilde{\Psi}_{x}, \widetilde{\Psi}_{y}$ and $\widetilde{\Psi}_{z}$ are given as:

$$
\left|\widetilde{\Psi}_{x}\right| \leq 2 \bar{H}_{0} \quad\left|\widetilde{\Psi}_{y}\right| \leq \bar{H}_{0} \quad\left|\widetilde{\Psi}_{z}\right| \leq \bar{H}_{0}|\sin i|
$$

$\bar{H}_{0}=\frac{\widetilde{H}_{0}}{H_{0}}$ can be found from Table I to $\bar{H}_{0} \approx 0.18$. Note that $\theta^{\prime}=\theta$ due to the circular orbit assumption. For an equatorial orbit, which is considered in this paper, the nominal B-field is constant with the only components out of the orbit plane, while the error term out-of-plane vanishes.

\section{NONLINEAR MODEL}

Introducing a state vector as $\mathbf{x}=\left[\begin{array}{llll}x_{1} & x_{2} & x_{3} & x_{4}\end{array}\right]^{T}=$ $\left[\begin{array}{llll}\alpha & \dot{\alpha} & \beta & \dot{\beta}\end{array}\right]^{T}$ a state space description of the system can be written as:

$$
\dot{\mathbf{x}}=\mathbf{f}(\mathbf{x})+\mathbf{g}(\mathbf{x}) u .
$$

To simplify the model, the control input is introduced as $u=\Lambda \frac{R_{E}^{3} H_{0}}{R_{B}^{3}} I$, which is a dimensionless quantity proportional to the current through the tether. The functions of the nonlinear state space description can be defined as:

$$
\begin{aligned}
& \mathbf{f}(\mathbf{x})= {\left[\begin{array}{c}
x_{2} \\
2\left(1+x_{2}\right) x_{4} \tan x_{3}-\frac{3}{2} \sin 2 x_{1} \\
x_{4} \\
-\frac{1}{2}\left(\left(1+x_{2}\right)^{2}+3 \cos ^{2} x_{1}\right) \sin 2 x_{3}
\end{array}\right] } \\
& 0 \\
& \mathbf{g}(\mathbf{x})=
\end{aligned}
$$

From the nominal system description $\left(\widetilde{\Psi}_{x}=\widetilde{\Psi}_{y}=0\right)$ it is seen that all equilibrium points are placed in the orbit plane, furthermore the system is only stabilizable under constant current in the orbit plane. The in-plane tether motion corresponds to the motion of a forced pendulum of angle $2 x_{1}$

$$
\begin{aligned}
& \dot{x}_{1}=x_{2} \\
& \dot{x}_{2}=-\frac{3}{2} \sin 2 x_{1}-u .
\end{aligned}
$$

The equilibrium points of the unforced system $(u=0)$ are the vertical tether positions $\left(x_{1}=0^{\circ}\right.$ and $\left.x_{1}=180^{\circ}\right)$ and the horizontal tether positions $\left(x_{1}= \pm 90^{\circ}\right)$. The vertical equilibria are stable, while the horizontal ones are unstable.

\section{CONTROL DESIGN}

In the following sections three controllers are designed to stabilize the in-plane tether position. First a linear controller is designed as basis for the evaluation of the nonlinear controllers. Second a controller is designed based on the feedback linearized system, to decouple the motion of the internal dynamics (the out-of-plane motion) from the in-plane motion. Last a sliding mode controller is designed to provide robustness against the uncertainties in the magnetic field.

\section{A. $L Q$ controller}

In a linear approximation around the vertical equilibrium $\left(x=\left[\begin{array}{lll}0 & 0 & 0\end{array}\right]^{T}\right)$ the nominal system can be written as:

$$
\dot{\mathbf{x}}=\left[\begin{array}{cccc}
0 & 1 & 0 & 0 \\
-3 & 0 & 0 & 0 \\
0 & 0 & 0 & 1 \\
0 & 0 & -4 & 0
\end{array}\right] \mathbf{x}+\left[\begin{array}{c}
0 \\
-1 \\
0 \\
0
\end{array}\right] u
$$


It is seen that the motions in and out of the orbit plane are decoupled and that the motion out of the plane is uncontrollable. The out-of-plane motions are uncontrollable since the B-field is perpendicular to the orbit plane, hence a Lorentz force can only be generated in the orbit plane. The LQ controller is designed for the controllable subspace of the system, while the remaining states are ignored, i.e. a linear control law is choosing as

$$
u=-k_{1} x_{1}-k_{2} x_{2} .
$$

\section{B. Feedback linearization}

In this section, the nominal system is feedback linearized to cancel the influence of the out-of-plane motion in the in-plane motion. The linearization is based on [8]. Similar to the linear case where the out-of-plane motions were uncontrollable, it can be shown that the system is not full-state linearizable in the orbit plane, hence the out-ofplane motion is considered internal dynamics by choosing the output as the in-plane angle $y=h(\mathbf{x})=x_{1}$. The system can be written in normal form without redefining the states

$$
\begin{aligned}
\dot{\boldsymbol{\eta}} & =\mathbf{f}_{0}(\boldsymbol{\eta}, \boldsymbol{\xi}) \\
\dot{\boldsymbol{\xi}} & =\mathbf{A}_{c} \boldsymbol{\xi}+\mathbf{B}_{c} \bar{\gamma}(\mathbf{x})(u-a(\mathbf{x})) \\
y & =\mathbf{C}_{c} \boldsymbol{\xi}
\end{aligned}
$$

where $\boldsymbol{\eta}=\left[\begin{array}{ll}x_{3} & x_{4}\end{array}\right]^{T}$ is the state vector associated with the out-of-plane motion, while $\mathbf{f}_{0}$ represent the internal dynamics of the system. $\boldsymbol{\xi}=\left[x_{1} x_{2}\right]^{T}$ is the state vector connected with the in-plane motion, while $\left(\mathbf{A}_{c}, \mathbf{B}_{c}, \mathbf{C}_{c}\right)$ represent a canonical state space description, i.e. a row of two integrators. The functions $a(\mathbf{x})$ and $\bar{\gamma}(\mathbf{x})$ can be found using the Lie derivative as:

$$
\begin{aligned}
a(\mathbf{x}) & =-\frac{L_{f}^{2} h(\mathbf{x})}{L_{\bar{g}} L_{f} h(\mathbf{x})} \\
& =2\left(1+x_{2}\right) x_{4} \tan x_{3}-\frac{3}{2} \sin 2 x_{1} \\
\bar{\gamma}(\mathbf{x}) & =L_{\bar{g}} L_{f} h(\mathbf{x})=-1
\end{aligned}
$$

where $\bar{g}$ indicates that the derivative is taken w.r.t. the nominal system. The system can be input-output linearized by choosing the control law

$$
u=a(\mathbf{x})+b(\mathbf{x}) v
$$

where $b(\mathbf{x})=\bar{\gamma}^{-1}(\mathbf{x})$ and $v$ is the new input. The resulting system describes the in-plane motion in canonical form. The zero dynamics of the system is defined as $\dot{\boldsymbol{\eta}}=\mathbf{f}_{0}(\boldsymbol{\eta}, 0)$, which can be shown to by stable, but not asymptotically stable. The lack of asymptotically stable zero dynamics prevent stability conclusions to be drawn for the entire closed loop system. The controller designed for the feedback linearized system is an LQ controller, using the same weights as the linear controller described in the previous section.

\section{Sliding mode}

In this section a sliding mode controller is designed with the aim to provide robustness to the uncertainties in the B-field. The sliding mode control design is based on the system in normal form (18), hence only the external dynamics is controlled. First a linear sliding surface is defined in state space as:

$$
s=k x_{1}+x_{2}=0 .
$$

Since the system is in normal form $x_{2}=\dot{x}_{1}$, hence the system behavior at the manifold is asymptotically stable for $k>0$. The next step is to design a control law which will lead every trajectory of the system onto the surface. This is done based on a quadratic Lyapunov candidate $V=\frac{1}{2} s^{2}$. The derivative can be written as $\dot{V}=s \dot{s}$, hence the control law must be designed such that $\dot{s}$ has the opposite sign of $s$ for $s \neq 0 . \dot{s}$ can be written in terms of the Lie derivatives of the system

$$
\dot{s}=k x_{2}+L_{f}^{2} h(\mathbf{x})+L_{g} L_{f} h(\mathbf{x}) u .
$$

The derivative $L_{f}^{2} h(\mathbf{x})$ is similar to the one found during the feedback linearization of the system, since no uncertainties are introduced in $\mathbf{f}(\mathbf{x})$, whereas $L_{g} L_{f} h(\mathbf{x})$ includes the uncertainties

$$
\begin{aligned}
\gamma(\mathbf{x}) & =L_{g} L_{f} h(\mathbf{x}) \\
& =\tan x_{3}\left(\widetilde{\Psi}_{x} \cos x_{1}+\widetilde{\Psi}_{y} \sin x_{1}\right)-1 .
\end{aligned}
$$

The control law is chosen based on the nominal system

$$
u=-\frac{1}{\bar{\gamma}(\mathbf{x})}\left(k x_{2}+L_{f}^{2} h(\mathbf{x})\right)+v
$$

where $v$ is a new control input. Note that the control law leads to $\dot{s}=\bar{\gamma}(\mathbf{x}) v$ assuming a nominal system, while it for the system with uncertainties can be written

$$
\dot{s}=\gamma(\mathbf{x}) v+\underbrace{\left(k x_{2}+L_{f}^{2} h(\mathbf{x})\right)\left(1-\frac{\gamma(\mathbf{x})}{\bar{\gamma}(\mathbf{x})}\right)}_{\Delta(\mathbf{x})}
$$

where $\Delta(\mathbf{x})$ is the part introduced due to the uncertainties. In the general case $v$ can not be chosen to ensure $\dot{s}$ to have the opposite sign of $s$, since $\gamma(\mathbf{x})$ is not upper bounded (the upper bound is of interest since $\bar{\gamma}(\mathbf{x})<0$ ). However assuming that $\left|x_{3}\right| \leq 45^{\circ}$, the following inequality can be stated:

$$
\gamma(\mathbf{x})<-1+\sqrt{\bar{\Psi}_{x}^{2}+\bar{\Psi}_{y}^{2}}
$$

where $\bar{\Psi}_{x}=2 \bar{H}_{0}$ and $\bar{\Psi}_{y}=\bar{H}_{0}$ are the boundary of the uncertainties found in Section III. Since $\gamma(\mathbf{x})<0$, the discontinuous control law $v=\kappa(\mathbf{x}) \operatorname{sgn}(s)$, where $\operatorname{sgn}$ denotes the sign function is chosen. Applying the control law

$$
\dot{s}=\gamma(\mathbf{x}) \kappa(\mathbf{x}) \operatorname{sgn}(s)+\Delta(\mathbf{x}) .
$$

$\kappa(\mathbf{x})$ is the controller gain which must be chosen such that $\kappa(\mathbf{x}) \gamma(\mathbf{x})$ dominates $\Delta(\mathbf{x})$, i.e.

$$
\begin{aligned}
\kappa(\mathbf{x}) & >\left|\frac{\Delta(\mathbf{x})}{\gamma(\mathbf{x})}\right| \\
& >\left|k x_{2}+L_{f}^{2} h(\mathbf{x})\right|\left|\frac{1}{\gamma(\mathbf{x})}-\frac{1}{\bar{\gamma}(\mathbf{x})}\right| \\
& >\left|k x_{2}+L_{f}^{2} h(\mathbf{x})\right| \frac{\sqrt{\bar{\Psi}_{x}^{2}+\bar{\Psi}_{y}^{2}}}{1-\sqrt{\bar{\Psi}_{x}^{2}+\bar{\Psi}_{y}^{2}}}
\end{aligned}
$$


To fulfill the inequality the gain is chosen as

$$
\kappa(\mathbf{x})=\left|k x_{2}+L_{f}^{2} h(\mathbf{x})\right| \frac{\sqrt{\bar{\Psi}_{x}^{2}+\bar{\Psi}_{y}^{2}}}{1-\sqrt{\bar{\Psi}_{x}^{2}+\bar{\Psi}_{y}^{2}}}+\kappa_{0}
$$

where $\kappa_{0}>0$. Stability of the in-plane motions can be guaranteed under the stated uncertainties and for $\left|x_{3}\right|<$ $45^{\circ}$, but overall stability can not be stated due to the zero dynamics of the system. The condition $\left|x_{3}\right|<45^{\circ}$ is actually quite critical, since $x_{3}$ is a part of the internal dynamics. It is, however, natural since the influence of the B-field component out of the orbit plane (the nominal B-field) will decrease with the out-of-plane angle.

\section{Simulations}

In this section the controller designs are validated through simulations. The control objective is to stabilize the in plane tether position at $x_{1}^{*}=30^{\circ}$, corresponding to a steady state input of $u=-\frac{3 \sqrt{3}}{4}$. To test the influence of the out-of-plane motions on the controller performance, these are excited by an initial displacement of $30^{\circ}$. To investigate the robustness due to the B-field uncertainties, the simulations are carried out both using a nontilted dipole and a tilted dipole. The main-satellite is assumed to have a orbit period of $90 \mathrm{~min}$. which allows the rotation time of the Earth to be formulated as $T_{E}=32 \pi$ w.r.t the dimensionless time. The control parameters of the three controllers can be seen in Table II.

TABLE II

CONTROL PARAMETERS USED IN SIMULATIONS

\begin{tabular}{|c|c|r|}
\hline Controller & Parameter & Value \\
\hline \hline \multirow{2}{*}{ LQ } & $k_{1}$ & -5.73 \\
\cline { 2 - 3 } & $k_{2}$ & -4.63 \\
\hline \multirow{2}{*}{$\begin{array}{c}\text { LQ for FB } \\
\text { linearized system }\end{array}$} & $k_{1}$ & 7.07 \\
\cline { 2 - 3 } Sliding mode & $k_{2}$ & 4.91 \\
\cline { 2 - 3 } & $k$ & 1.00 \\
\hline
\end{tabular}

\section{A. In-plane motions}

The simulation of the LQ controller is shown in Fig. 3. The design of the LQ controller is based on a linearization at $x_{1}^{*}=30^{\circ}$, hence no steady state error occur. It is seen that both simulation fails to stabilize the position during the 5 orbits. The variation seems worse in the simulation with the tilted dipole, which occur since uncertainties are introduced both through the B-field and through the internal dynamic. Fig. 4 shows the simulation of the feedback linearized system. In contrast to the linear controller the out-of-plane motion is totally decoupled from the in-plane motion. The uncertainties in the Bfield have an influence on the in-plane motion, however not as much as for the linear controller. The simulation of the sliding mode controller (Fig. 5) with the nominal system (i.e. the nontilted dipole) shows a fine ability to stabilize the in-plane position. Also the disturbance rejection is quite good. It is seen that the out-of-plane angle actually exceeds the $45^{\circ}$ limit, this has however no critical influence on the performance in this case.
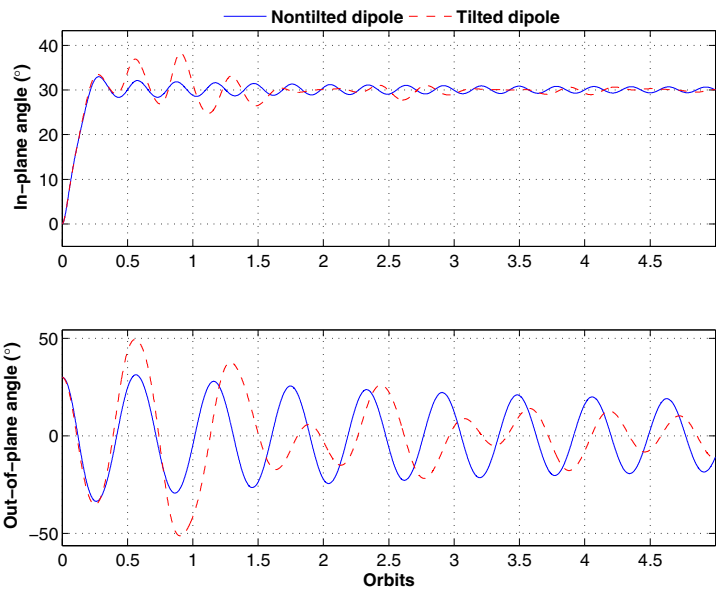

Fig. 3. Closed loop simulation with the LQ controller. The internal dynamics affect the in-plane motion in the simulation with both dipole models.
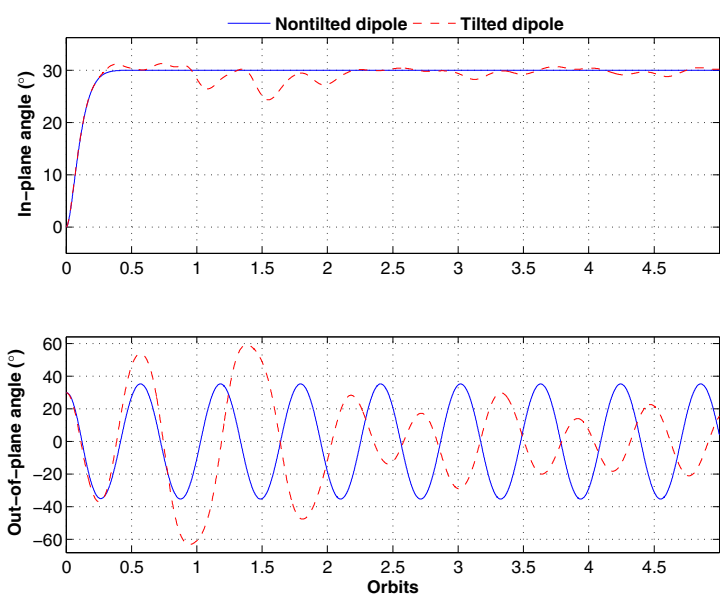

Fig. 4. Closed loop simulation with the LQ controller based on the feedback linearized system. The internal dynamic is totally decoupled in the nominal case, but not when uncertainties are introduced in the magnetic field.

\section{B. Out-of-plane motions}

None of the controllers are designed to control the out-of-plane motion, since in the linear approach, it is uncontrollable and in the feedback linearization it is internal dynamics. The response of the internal dynamic is, however, important in connection with both the overall stability and with the robustness of the controllers. Fig. 6 shows the response of the out-of-plane motion, for a simulation with the nominal system. The simulation time is 35 orbits. The out-of-plane motion is damped by the LQ controller, while it is not affected by the remaining two controllers. This is in contrast to the in-plane stabilization, where the LQ controller had the poorest performance. This phenomenon can be explained by the energy function of the system. The total energy can be written in a nondimensional form as [2]:

$$
\mathcal{E}=\frac{1}{2}\left(4+x_{4}^{2}-\cos ^{2} x_{3}\left(1-x_{2}^{2}+3 \cos ^{2} x_{1}\right)\right) .
$$



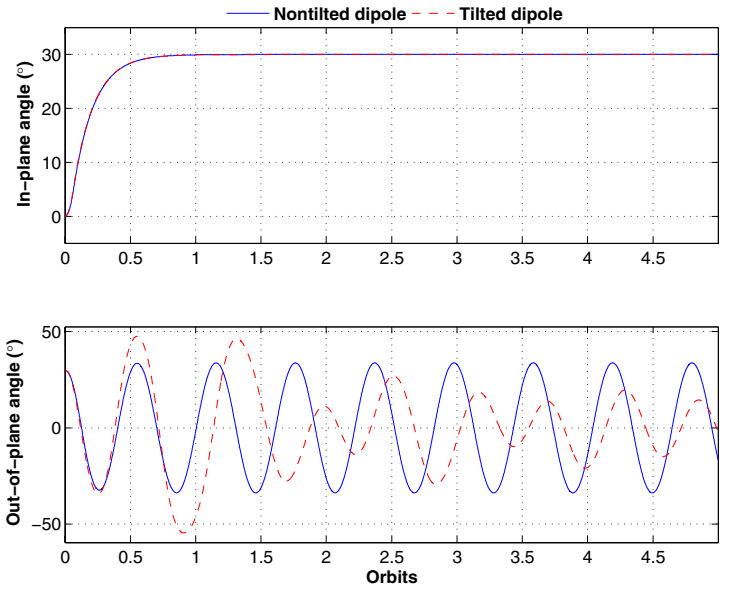

Fig. 5. Closed loop simulation with the sliding mode controller. It is seen that the robustness w.r.t. the uncertainties in the magnetic field is increased compared to the other controllers.
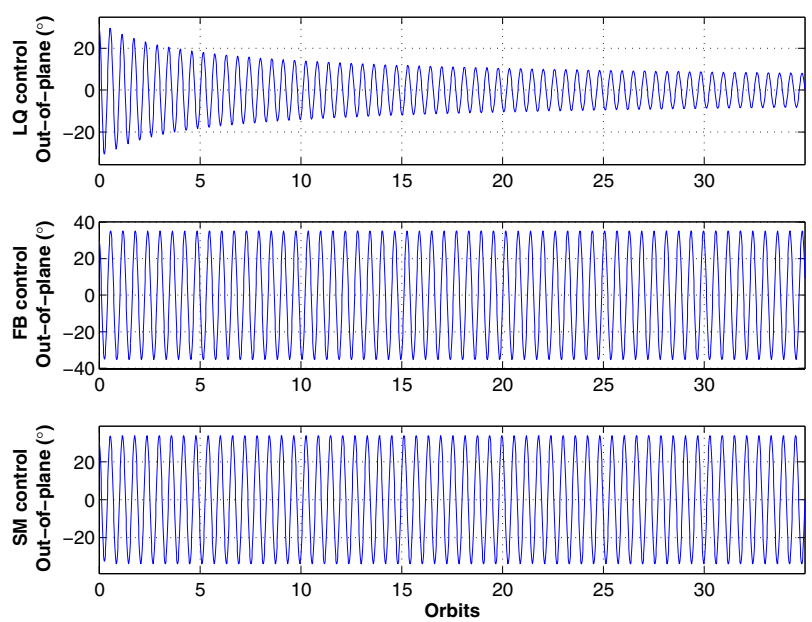

Fig. 6. Out-of-plane angle with nontilted dipole. The LQ controller damps the out-of-plane motions, in contrast to the other controllers.

The change of energy in the system can be found as:

$$
\begin{array}{r}
\frac{d \mathcal{E}}{d \tau}=x_{2} \cos ^{2} x_{3} \\
\left(\tan x_{3}\left(\widetilde{\Psi}_{x} \cos x_{1}+\widetilde{\Psi}_{y} \sin x_{1}\right)-1\right) u+ \\
x_{4}\left(\widetilde{\Psi}_{y} \cos x_{1}-\widetilde{\Psi}_{x} \sin x_{1}\right) u .
\end{array}
$$

For the nominal system, no change in the energy will occur when the in-plane motions is stabilized, i.e. $x_{2}=0$. Fig. 7 shows a similar simulation with the tilted dipole model. It is seen that the out-of-plane motion is damped by all three controllers. The motion is damped to a level where only a sequence with same period as the rotation of the Earth is present. However, simulations has shown that this property depends on the in-plane operation point and that the internal dynamics could be driven unstable when the tether position is stabilized at $x_{1}^{*}<0$. This corresponds to a steady state input $u>0$, where operation of the tether is altered from generator mode (decreasing the altitude) to thruster mode (increasing the altitude).
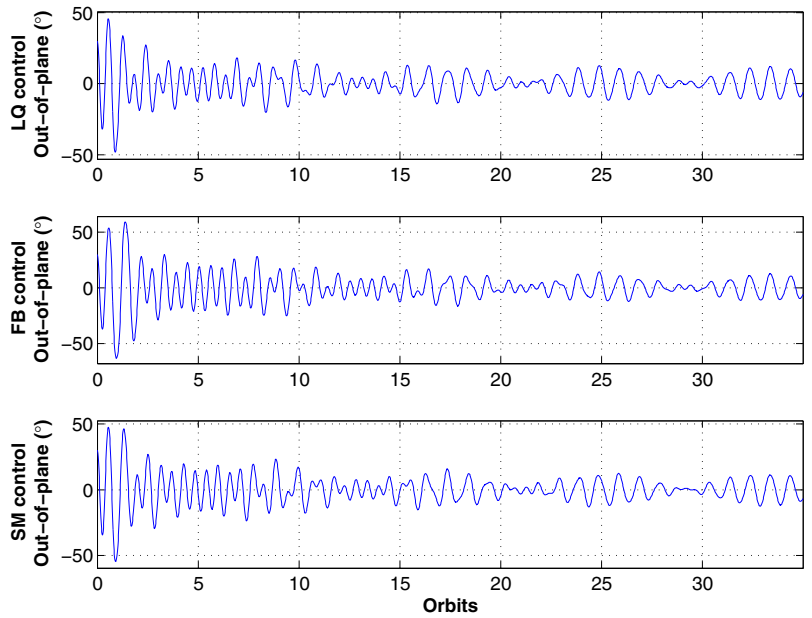

Fig. 7. Out-of-plane motion with tilted dipole. It is seen that the internal dynamics is damped by the uncertainties in the magnetic field.

\section{Discussion}

The controllers presented in this paper are all providing acceptable performance for the in-plane motion, however, to expand the stabilization to the out-of-plane angle other design methods must be used, e.g. energy based methods. This is necessary since the out-of-plane motion is uncontrollable and its stability depends on the uncertainties.

\section{CONCLUSION}

This paper designed a feedback linearization for a tethered satellite system in a near-equatorial orbit, to stabilize the in-plane tether position, while decouple the out-ofplane dynamics. Furthermore, a sliding mode controller was designed to achieve robustness to uncertainties in the magnetic field and stability was shown for the inplane dynamics under ideal conditions. The feedback linearization was shown to reduce coupling of out-ofplane motion to the external dynamics, however with the consequence that any initial out-of-plane motion remained undamped. Taking the uncertainties in the B-field into account, simulations showed how the stability of the internal dynamics depends on the in-plane operation point.

\section{REFERENCES}

[1] V. V. Beletsky and E. M. Levin, Dynamics of space tether systems. American Astronautical society, 1993.

[2] O. Lopez-Rebollal, J. Pelaez, E. Lorenzini, and M. Ruiz, "A new kind of dynamic instability in electrodynamic tethers," Journal of the Astronautical Sciences, vol. 48, no. 4, pp. 449-476, 2000.

[3] J. Corsi and L. Iess, "Stability and control of electrodynamic tethers for de-orbiting applications," Acta Astronautica, vol. 48, no. 5-12, pp. 491-501, 2001.

[4] J. Pelaez and E. Lorenzini, "Libration control of electrodynamic tethers in inclined orbit," Journal of Guidance, Control, and Dynamics, vol. 28, no. 2, pp. 269-279, 2005.

[5] P. Williams, "Energy rate feedback for libration control of electrodynamic tethers," Journal of Guidance, Control, and Dynamics, vol. 29, no. 1, pp. 221-223, 2006.

[6] K. K. Mankala and S. K. Agrawal, "Equilibrium-to-equilibrium maneuvers of rigid electrodynamic tethers," Journal of Guidance, Control, and Dynamics, vol. 28, no. 3, pp. 541-545, 2005.

[7] S. Macmillan and S. Maus, "International geomagnetic reference field - the tenth generation," Earth, Planets and Space, vol. 57, no. 12 , pp. 1135-1140, 2005.

[8] H. K. Khalil, Nonlinear Systems, 3rd ed. Prentice Hall, 2002. 